\title{
A New Xanthone from the Bark Extract of Rheedia acuminata and Antiplasmodial Activity of Its Major Compounds
}

\author{
Guillaume Marti ${ }^{1}$, Véronique Eparvier ${ }^{2, *}$, Marc Litaudon ${ }^{1}$, Philippe Grellier ${ }^{3}$ and \\ Françoise Guéritte ${ }^{1}$
}

1 Centre de Recherche de Gif, Institut de Chimie des Substances Naturelles, CNRS, 1 avenue de la Terrasse, 91198 Gif-sur-Yvette Cedex, France; E-Mails: guillaume.marti@icsn.cnrs-gif.fr (G.M.); marc.litaudon@icsn.cnrs-gif.fr (M.L.); francoise.gueritte@icsn.cnrs-gif.fr (F.G.)

2 UPS2561, CNRS, 2, avenue Gustave Charlery, 97300 Cayenne, France

3 Muséum National d'Histoire Naturelle, FRE 3206 CNRS, CP52, 61 rue Buffon, 75231 Paris cedex 05, France; E-Mail: grellier@mnhn.fr (P.G.)

* Author to whom correspondence should be addressed; E-Mail: veronique.eparvier@guyane.cnrs.fr; Tel.: +594-594-29-75-16; Fax: +594-594-28-47-86.

Received: 21 August 2010; in revised form: 23 September 2010 / Accepted: 3 October 2010 / Published: 14 October 2010

\begin{abstract}
Bioassay-guided fractionation of the ethyl acetate bark extract of Rheedia acuminata led to the isolation of the new compound 1,5,6-trihydroxy-3-methoxy-7geranyl-xanthone (1), together with four known compounds 2-5. These compounds were tested in vitro for their antiplasmodial activity on a chloroquine-resistant strain of Plasmodium falciparum (FcB1) and for their cytotoxicity against the human diploid embryonic lung cell line MRC-5.
\end{abstract}

Keywords: Rheedia acuminata, Clusiaceae; xanthones; antiplasmodial activity; cytotoxicity

\section{Introduction}

In South America several species of Clusiaceae are widely used in the manufacture of hulls, and well known for quality of their wood and for the healing properties of their latex used traditionally used for their effectiveness against dermatoses [1]. One of these, Rheedia acuminata (Ruiz \& Pavon) Planchon and Triana, a tree growing in Amazonian rainforest, possesses an abundant latex used for 
various medicinal purposes by the Guianese Amerindians (Palikur) in the form of patches or breakdowns applied to the wrinkling muscle. In addition, Rheedia acuminata fruits are commonly consumed in South America [1].

Prenylated xanthones and polyprenylated acylphloroglucinols (PPAPs) are widely distributed in the Clusiaceae, the genus Rheedia being a rich source of them [2-5]. Some biflavonoids were also previously isolated from this species [6]. Their biological activities include antibacterial, analgesic and cytotoxic properties $[5,7,8]$.

In an effort to find new natural antimalarial drugs and as part of investigation of French Guiana plants, we found that the ethyl acetate extract of the bark of Rheedia acuminata showed antiplasmodial activity (92\% of inhibitory growth of Plasmodium falciparum (FcB1) at $10 \mu \mathrm{g} / \mathrm{mL}$ ), whereas leaves and fruits extracts showed no significant and weak activity respectively (28\% and $50 \%$ of inhibitory growth at $10 \mu \mathrm{g} / \mathrm{mL}$, respectively). We report here the bioassay-guided fractionation of this extract on the basis of this antiplasmodial activity.

\section{Results and Discussion}

The bioguided fractionation of the ethyl acetate extract of trunk bark of Rheedia acuminata led to the isolation of the new 1,5,6-trihydroxy-3-methoxy-7-geranyl-xanthone (1) along with two xanthone analogues (2 and 3) and two PPAPs (4 and 5) (Figure 1).

Figure 1. Structures of compounds 1-5.

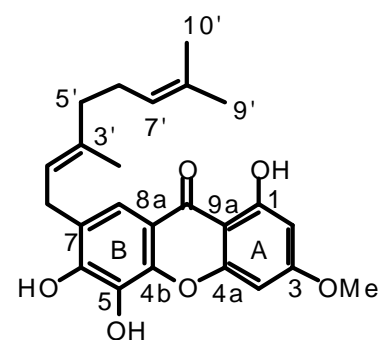

1<smiles>C=CC(C)(C)c1cc(O)c2c(c1O)C(=O)C1C=CC=C(O)C1O2</smiles>

2<smiles></smiles>

3
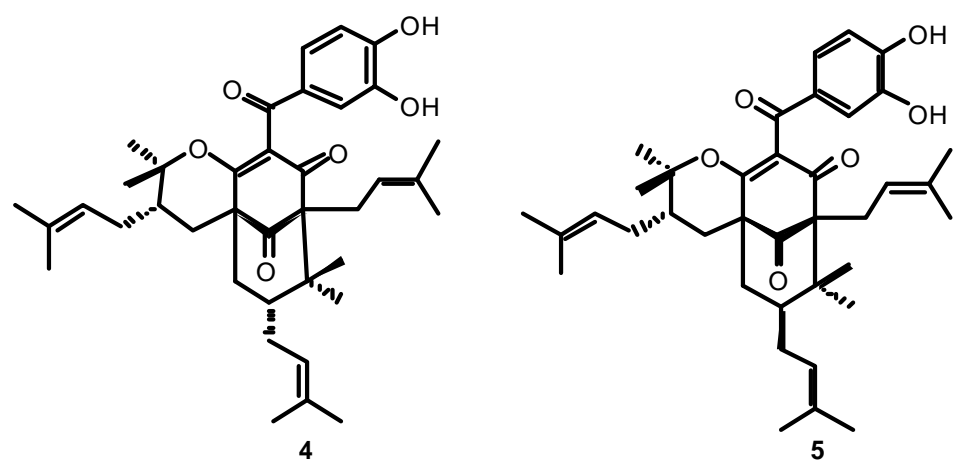

Compound 1 was obtained as a yellow oil. The HREIMS indicated a $[\mathrm{M}+\mathrm{H}]^{-}$ion peak at $\mathrm{m} / z$ 409.1652, giving the molecular formula $\mathrm{C}_{24} \mathrm{H}_{25} \mathrm{O}_{6}$ (calc. 409.1651). The IR spectrum displayed free hydroxyl $\left(3,410 \mathrm{~cm}^{-1}\right)$, chelated hydroxyl $\left(3,170 \mathrm{~cm}^{-1}\right)$, conjugated carbonyl $\left(1,640 \mathrm{~cm}^{-1}\right)$ and aromatic ring $\left(1,570 \mathrm{~cm}^{-1}\right)$ peaks. These data, together with absorption bands observed at $\lambda_{\max } 251,283$ and 327 $\mathrm{nm}$ in the UV spectrum and those obtained from 1D and 2D NMR experiments of compound $\mathbf{1}$ 
suggested the presence of a 1,3,5,6-tetraoxygenated xanthone system [10,11]. Examination of the ${ }^{1} \mathrm{H}$ - NMR spectrum showed the presence of three hydroxyl groups with two broad signals at $\delta_{\mathrm{H}} 7.88$, 12.68 and one singlet at $\delta_{\mathrm{H}} 14.02 \mathrm{ppm}$, corresponding to a chelated proton, one singlet at $\delta_{\mathrm{H}} 8.08$, two meta-coupled protons at $\delta_{\mathrm{H}} 6.56$ and $6.04(1 \mathrm{H}, \mathrm{d}, J=2.3 \mathrm{~Hz})$, and one methoxyl $\left(\delta_{\mathrm{H}} 3.63\right)$. In addition two vinyl protons at $\delta_{\mathrm{H}} 5.77$ and $5.26(1 \mathrm{H}, b r t, J=6.8 \mathrm{~Hz})$, three methylene groups at $\delta_{\mathrm{H}} 3.83(2 \mathrm{H}, d$, $J=7.3 \mathrm{~Hz}), 2.21(2 \mathrm{H}, m)$ and $2.15(2 \mathrm{H}, m)$, and three methyl groups at $\delta_{\mathrm{H}} 1.84,1.69,1.59(3 \mathrm{H}, s)$ suggested the presence of a geranyl side chain. HSQC experiments allowed the assignment of all protonated carbons. A combination of HMBC, COSY and NOESY experiments were used to establish the position of the substituents. In the HMBC spectrum (Figure 2), the chelated proton $\mathrm{OH}-1$ showed correlations with C-2 at $\delta_{\mathrm{c}} 97.6$ on one hand, and the proton H-2 with carbons C-9a $\left(\delta_{\mathrm{C}} 104.2\right), \mathrm{C}-4$ $\left(\delta_{\mathrm{C}} 92.8\right), \mathrm{C}-1\left(\delta_{\mathrm{C}} 164.7\right)$ and $\mathrm{C}-3\left(\delta_{\mathrm{C}} 166.7\right)$ on the other hand. A methoxy group was deduced from the correlation between the methyl protons at $\delta_{\mathrm{H}} 3.63$ with C-3. NOESY correlations between the methoxy at C-3 with protons $\mathrm{H}-2\left(\delta_{\mathrm{H}} 6.56\right)$ and $\mathrm{H}-4\left(\delta_{\mathrm{H}} 6.04\right)$ confirmed the position of the substituents on ring A (Figure 2). Furthermore, HMBC correlations observed between H-8 and C-9 $\left(\delta_{\mathrm{C}}\right.$ 181.5) were indicative of a peri location of the carbonyl. In addition, proton $\mathrm{H}-8$ showed correlations with C-6 $\left(\delta_{\mathrm{C}} 153.4\right), \mathrm{C}-4 \mathrm{~b}\left(\delta_{\mathrm{C}} 146.6\right)$, and $\mathrm{CH}_{2}-1$ ' $\left(\delta_{\mathrm{C}} 29.5\right)$ and a long-range correlation with $\mathrm{C} 9 \mathrm{a}\left(\delta_{\mathrm{C}} 104.2\right)$. The geranyl side chain was deduced from the COSY spectrum with, on one hand, correlations observed between Me-9' and Me-10' [ $\delta_{\mathrm{H}} 1.59$ (3H, s, H-9') and $\left.1.69\left(3 \mathrm{H}, s, \mathrm{H}-10^{\prime}\right)\right]$ and the vinylic proton $\mathrm{H}_{-1}{ }^{\prime}$ at $\delta_{\mathrm{H}} 5.28(1 \mathrm{H}, \mathrm{brt}, J=6.8 \mathrm{~Hz})$, which in turn showed a cross peak with $\mathrm{H}_{2}-6^{\prime}\left(\delta_{\mathrm{H}} 2.21,2 \mathrm{H}, m\right)$. On the other hand, correlations observed between $\mathrm{H}_{2}-5^{\prime}\left(\delta_{\mathrm{H}} 2.16,2 \mathrm{H}, m\right)$ and $\mathrm{H}_{2}-6$ ' and $\mathrm{H}-2$ ' at $\delta_{\mathrm{H}} 5.77(1 \mathrm{H}, b r t, J=6.8 \mathrm{~Hz})$, which in turn showed correlations with Me-4' $\left(\delta_{\mathrm{H}} 1.84\right.$, $3 \mathrm{H}, s)$ and $\mathrm{H}_{2}-1$ ' $\left(\delta_{\mathrm{H}} 3.83,2 \mathrm{H}, d, J=7.3 \mathrm{~Hz}\right)$ confirmed the presence of a geranyl side chain. The $E$ configuration of the C-2'-C-3' double bond was established by NOESY correlations observed between $\mathrm{H}_{2}-5$ ' with H-2', and Me-4'with $\mathrm{H}_{2}-1$ ' (Figure 2). Finally, the position of the geranyl side chain at C-7 $\left(\delta_{\mathrm{C}} 127.8\right)$ was confirmed by HMBC correlation from $\mathrm{H}_{2}-1$ ' to $\mathrm{C}-6\left(\delta_{\mathrm{c}} 153.4\right)$ (Figure 2). Compound $\mathbf{1}$, which was named 1,5,6-trihydroxy-3-methoxy-7-geranylxanthone, is a positional isomer of cowaxanthone and rubraxanthone isolated from Garcinia cowa $[12,13]$.

Figure 2. Key COSY (bold), HMBC (plain arrows) and NOESY (dashed arrows) correlations for $\mathbf{1}$.

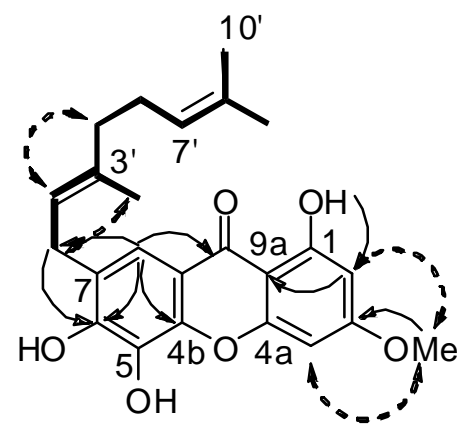

Compound 2 was identified as 2-(1'-1'-dimethylprop-2'-enyl)-1,4,5-trihydroxyxanthone and compound 3 as pyrojacareubin by comparison of the UV, HRESIMS, 1D and 2D NMR with the 
literature data [13-15]. The 1D and 2D NMR spectra associated with their optical rotation values confirmed that 4 was isogarcinol [16-18] and 5, 7-epi-isogarcinol [16].

The antiplasmodial activity against the chloroquine-resistant strain of $P$. falciparum $\mathrm{FcB} 1$ and the cytotoxicity upon the human diploid embryonic cell line MRC-5 of compounds 1-5 are summarized in Table 1. The three xanthones 1, 2 and 3 isolated from Rheedia acuminata bark have $\mathrm{IC}_{50} \mathrm{~S}$ against Plasmodium falciparum exceeding $10 \mu \mathrm{M}$. Compounds 4 and 5 have shown the best activity with $\mathrm{IC}_{50} \mathrm{~S}$ around $3 \mu \mathrm{M}$. The $\mathrm{IC}_{50}$ values obtained on MRC-5 cell line indicated that PPAPs 4 and 5 exhibited significant cytotoxicity whereas xanthones (1 to 3 ) showed weak cytotoxicity. Several xanthones, which have been isolated from various species of the family Clusiaceae, showed cytotoxic and antiplasmodial activities $[9,10,19,20]$ and structure-activity relationships have been proposed in this series. For example, Winter et al. have demonstrated that xanthones with a hydroxyl group in peri positions with regards to the carbonyl (such as 1, 2 and 3), possess weak antiplasmodial activity due to their low affinity for the heme [21,22]. In conclusion, the antiplasmodial activity detected in the crude extract is probably due to the presence of high quantity of the two PPAPs $\mathbf{4}$ and $\mathbf{5}$.

Table 1. $\mathrm{IC}_{50}$ values of compounds 1-5 tested against $\mathrm{FcB} 1$ strain of $P$. falciparum and MRC-5 cell.

\begin{tabular}{ccc}
\hline Compound & IC $_{\mathbf{5 0}} \boldsymbol{P . f .} \mathbf{F C B 1}(\boldsymbol{\mu M}) \pm$ SD $(\mathbf{n}=\mathbf{3})$ & IC $_{\mathbf{5 0}}$ MRC-5 $(\boldsymbol{\mu M}) \pm$ SD $(\mathbf{n}=\mathbf{3})$ \\
\hline $\mathbf{1}$ & $10.5^{\mathrm{a}}$ & $36^{\mathrm{a}}$ \\
$\mathbf{2}$ & $15.1^{\mathrm{a}}$ & $\mathrm{b}$ \\
$\mathbf{3}$ & $11.4^{\mathrm{a}}$ & $29^{\mathrm{a}}$ \\
$\mathbf{4}$ & $3.5 \pm 1.1$ & $3.5 \pm 0.4$ \\
$\mathbf{5}$ & $3.2 \pm 1.3$ & $2.3 \pm 0.5$ \\
Chloroquine & $0.078 \pm 0.006$ & $31.5 \pm 4.5$ \\
Taxotere & &
\end{tabular}

\section{Experimental}

\subsection{General}

The NMR spectra were recorded on a Bruker $500 \mathrm{MHz}$ (Avance 500) or $300 \mathrm{MHz}$ (Aspect DPX $300 \mathrm{MHz}$ ) spectrometer. ESIMS were obtained on a Thermoquest Navigator mass spectrometer. HRESIMS were obtained on a ESI-TOF spectrometer (LCT; Waters). Kromasil analytical, semi-preparative and preparative C-18 columns $(250 \times 4.5 \mathrm{~mm} ; 250 \times 10 \mathrm{~mm}$ and $250 \times 21.2 \mathrm{~mm}$ I.D, $5 \mu \mathrm{M}$ Thermo ${ }^{\circledR}$ ) were used for preparative HPLC separations using a "Waters autopurification system" equipped with a sample manager (Waters 2767), a column fluidics organizer, a binary pump (Waters 2525), a UV-Vis diode array detector (190-600nm), Waters 2996) and PL-ELS 1000 ELSD detector Polymer laboratory. IR spectra were obtained on a Nicolet FTIR 205 spectrophotometer. The UV spectra were recorded on a Perkin-Elmer Lambda 5 spectrophotometer. Specific rotation was obtained in $\mathrm{CHCl}_{3}$ with a JASCO P-1010 polarimeter. Silica gel $60(35-70 \mu \mathrm{m})$ and analytical TLC plates (Si gel $60 \mathrm{~F}$ 254) were purchased from SDS (France). Polyamide DC 6 and polyamide cartridge were purchased from Macherey-Nagel (Chromabond PA, $1 \mathrm{~g}$ ). 


\subsection{Plant Material}

Trunk bark of R. acuminata were collected in French Guiana. A voucher specimen (PMF-258) was deposited in the Herbarium of Cayenne (French Guiana), and identified by M-F. Prévost (IRD).

\subsection{Extraction and Isolation}

Trunk bark $(370 \mathrm{~g})$ were extracted three times with EtOAc $(3 \times 1 \mathrm{~L})$ at $40{ }^{\circ} \mathrm{C}$ and 1,450 psi on a Zippertex ${ }^{\circledR}$ static high-pressure high-temperature extractor developed in the ICSN Pilot Unit. This extract $(10 \mathrm{~g})$ was filtered on polyamide. Filtered extract $(9.3 \mathrm{~g})$ was subjected to silica gel chromatography using heptane/EtOAc mixtures (100/0-0/100). According to their TLC profile, 10 fractions were obtained (F1-F10). Fraction F4 (1.1 g), which showed antiplasmodial activity, was subjected to a preparative $\mathrm{C}-18$ column using an isocratic mobile phase consisting of $\mathrm{ACN} / \mathrm{water}$ $+0.1 \%$ formic acid (flow rate $21 \mathrm{~mL} / \mathrm{min}$ ). This resulted in the isolation of $4(72 \mathrm{mg}, 0.019 \%)$, 5 (65 mg, 0.017\%), 3 (2 mg, 0.0008\%), 1 (8 mg, 0.0021\%) and 2 (18 mg, 0.004\%) with retention times of $15.5,13.5,10.4,9.4$, and 4.6 mins, respectively.

1,5,6-Trihydroxy-3-methoxy-7-geranylxanthone (1): Yellow oil; UV (MeOH) $\lambda_{\max }(\log \varepsilon)$ : 327 (3.99), 283 (3.96), 251 (4.35); IR $v_{\max }$ (ns) 3410, 1640, 1570, 1450, 1370, 1290, $1220 \mathrm{~cm}^{-1}$; ${ }^{1} \mathrm{H}-\mathrm{NMR}$ (pyridine- $\left.d_{5}, 500 \mathrm{MHz}\right): \delta 14.02(1 \mathrm{H}, s, \mathrm{OH}-1), 12.68(1 \mathrm{H}$, brs, $\mathrm{OH}), 8.08(1 \mathrm{H}, s, \mathrm{H}-8), 7.88(1 \mathrm{H}, b r s$, OH) $6.56(1 \mathrm{H}, d, J=2.3 \mathrm{~Hz}, \mathrm{H}-2), 6.04(1 \mathrm{H}, d, J=2.3 \mathrm{~Hz}, \mathrm{H}-4), 5.77(1 \mathrm{H}, b r t, J=6.8 \mathrm{~Hz}, \mathrm{H}-2$ '), 5.28 $\left(1 \mathrm{H}, b r t, J=6.8 \mathrm{~Hz}, \mathrm{H}-7^{\prime}\right), 3.83$ ( 2H, brd, $J=7.3 \mathrm{~Hz}, \mathrm{H}-1$ '), 3.63 (3H, s, OMe-3), 2.21 (2H, m, H-6'), $2.15\left(2 \mathrm{H}, m, \mathrm{H}-5\right.$ '), 1.84 (3H, $\left.s, \mathrm{H}-4^{\prime}\right), 1.69\left(3 \mathrm{H}, s, \mathrm{H}-10^{\prime}\right), 1.59$ (3H, $\left.s, \mathrm{H}_{-} 9^{\prime}\right) ;{ }^{13} \mathrm{C}-\mathrm{NMR}$ (pyridine- $d_{5}$, $125 \mathrm{MHz}$ ): $\delta 181.5$ (C-9), 166.7 (C-3), 164.7 (C-1), 158.5 (C-4a), 153.4 (C-6), 146.6 (C-4b), 137.2 (C3'), 133.9 (C-5), 131.8 (C-8'), 127.8 (C-7), 125.3 (C-7'), 123.5 (C-2'), 116.7 (C-8), 113.8 (C-8a),

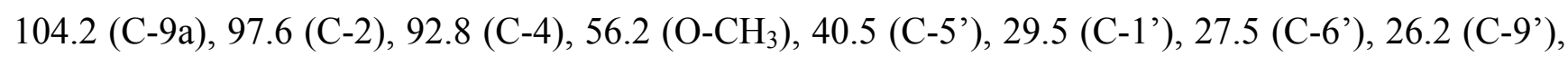
17.7 (C-4'), 18.2 (C-10'); HREIMS [M-H] ${ }^{-} m / z$ 409.1697, $\mathrm{C}_{24} \mathrm{H}_{26} \mathrm{O}_{6}$ requires 409.1651.

2-(1', 1'-Dimethylprop-2'-enyl)-1,4,5-Trihydroxyxanthone (2): Yellow powder; UV (MeOH) $\lambda_{\max }(\log$ ع): 320 (3.61), 263 (4.12), 249 (4.12), 236 (4.07); IR $v_{\max }$ (ns) 3570, 1720, 1680, 1540, 1270, $1120 \mathrm{~cm}^{-}$ ${ }^{1}$; ${ }^{1} \mathrm{H}-\mathrm{NMR}$ (pyridine- $\left.d_{5}, 500 \mathrm{MHz}\right): \delta 13.55(1 \mathrm{H}, s,-\mathrm{OH}), 7.99(1 \mathrm{H}, d d, J=8.0 \mathrm{~Hz}, \mathrm{H}-8), 7.72(1 \mathrm{H}, s$, $\mathrm{H}-3), 7.53(1 \mathrm{H}, d d, J=8.3,1.5 \mathrm{~Hz}, \mathrm{H}-6), 7.27(1 \mathrm{H}, t, J=8.0 \mathrm{~Hz}, \mathrm{H}-7), 6.47(1 \mathrm{H}, d d, J=17.1,10.4$ Hz, H-2'), 5.15 (2H, $m, \mathrm{H}-3$ ') 1.66 (6H, H-4' , H-5'); ${ }^{13} \mathrm{C}$ - NMR (pyridine- $\left.d_{5}, 125 \mathrm{MHz}\right): \delta 184.4(\mathrm{C}-$ 9), 153.2 (C-1), 149.7 (C-5), 148.7 (C-2'), 148.6 (C-4b), 144.2 (C-4a), 138.1 (C-4), 128.7 (C-2), 124.7 (C-3), 124.6 (C-7), 123.4 (C-8a), 122.2 (C-6), 116.1 (C-8), 112.3 (C-3'), 110.1 (C-9a), 41.1 (C-1'), 27.2 (C-4', C-5'); HREIMS [M-H] ${ }^{-} m / z$ 311,0934, $\mathrm{C}_{18} \mathrm{H}_{16} \mathrm{O}_{5}$ requires 311.0919.

Pyrojacareubine (3): Orange-yellow powder; UV (MeOH) $\lambda_{\max }(\log \varepsilon)$ : 329 (3.42), 276 (4,18), 220 (4.08); IR $v_{\max }$ (ns) 3330, 1650, 1580, 1490, 1450, 1290, $1220 \mathrm{~cm}^{-1}$; ${ }^{1} \mathrm{H}-\mathrm{NMR}$ (pyridine- $d_{5}, 500 \mathrm{MHz}$ ): $\delta 13.05(1 \mathrm{H}, s, 1-\mathrm{OH}), 7.46(1 \mathrm{H}, s, \mathrm{H}-8), 6.90(1 \mathrm{H}, d, J=10.0 \mathrm{~Hz}, \mathrm{H}-2$ '), $6.45(1 \mathrm{H}, d, J=10.1 \mathrm{~Hz}$, H-7'), 6.27 (1H, s, H-4), 5.74 ( $1 \mathrm{H}, d, J=10.1 \mathrm{~Hz}, \mathrm{H}-8$ '), 5.62 (1H, $d, J=10.0 \mathrm{~Hz}, \mathrm{H}-3$ ') 1.55 (6H, $s$, H-10', H-11'), 1.50 (6H, s, H-5', H-6'); ${ }^{\prime 3} \mathrm{C}^{-N M R}$ (pyridine- $d_{5}, 125 \mathrm{MHz}$ ): $\delta 180.8$ (C-9), 164.2 (C-1), 161.5 (C-3), 146.4 (C-4a), 131.0 (C-8'), 127.2 (C-3'), 121.4 (C-7'), 118.2 (C-7), 115.2 (C-2'), 113.5 
(C-8), 110.4 (C-8a), 103.3 (C-2), 101.6 (C-9a), 99.4 (C-4), 78.9 (C-9'), 78.2 (C-4'), 28.4 ( C-10', C-11'), 28.3 (C-5', C-6’); HREIMS [M-H] ${ }^{-} \mathrm{m} / z$ 391.1177, $\mathrm{C}_{23} \mathrm{H}_{19} \mathrm{O}_{6}$ requires 391.1182.

Isogarcinol (4): Brown powder; $[\alpha]_{\mathrm{D}}^{25}=-160\left(c=1.0, \mathrm{CHCl}_{3}\right) ; \mathrm{UV}(\mathrm{MeOH}) \lambda_{\max }(\log \varepsilon): 317(3.82)$, 277 (4,14), 233 (4.07); IR $v_{\max }$ (ns) 3290, 2920, 2850, 1730, 1670, 1590, 1520, 1440, 1370, 1290, 1170 $\mathrm{cm}^{-1}$; ${ }^{1} \mathrm{H}-\mathrm{NMR}$ (pyridine- $\left.d_{5}, 500 \mathrm{MHz}\right): \delta 8.05(1 \mathrm{H}, d, J=2.0 \mathrm{~Hz}, \mathrm{H}-12), 7.68(1 \mathrm{H}, d d, J=8.1,2.0$ $\mathrm{Hz}, \mathrm{H}-16), 7.28(1 \mathrm{H}, d, J=8.1 \mathrm{~Hz}, \mathrm{H}-15), 5.42(1 \mathrm{H}, t l, J=6.5 \mathrm{~Hz}, \mathrm{H}-18), 5.09(2 \mathrm{H}, t l, J=6.5 \mathrm{~Hz}$, H-35, H-25), $3.27(1 \mathrm{H}, d d, J=13.9,3.1 \mathrm{~Hz}, \mathrm{H}-29), 3.21(1 \mathrm{H}, d d d, J=14.4,10.7,9.5 \mathrm{~Hz}, \mathrm{H}-24), 2.76$ $(1 \mathrm{H}, d d, J=13.7,5.6 \mathrm{~Hz}, \mathrm{H}-17), 2.43(1 \mathrm{H}, d l, J=14.1 \mathrm{~Hz}, \mathrm{H}-8), 2.42(1 \mathrm{H}, d l, J=14.1 \mathrm{~Hz}, \mathrm{H}-24)$, $2.11(1 \mathrm{H}, d d, J=14.1,7.3 \mathrm{~Hz}, \mathrm{H}-8), 1.96(1 \mathrm{H}, d l, J=14.1 \mathrm{~Hz}, \mathrm{H}-34), 1.91(3 \mathrm{H}, s, \mathrm{H}-28), 1.82(1 \mathrm{H}$, $d d d, J=14.2,9.5,9.5 \mathrm{~Hz}, \mathrm{H}-34), 1.74(3 \mathrm{H}, s, \mathrm{H}-27), 1.71(3 \mathrm{H}, s, \mathrm{H}-21), 1.68(3 \mathrm{H}, s, \mathrm{H}-37), 1.66(1 \mathrm{H}$, $d t, J=9.9,5.0 \mathrm{~Hz}, \mathrm{H}-30), 1.57(3 \mathrm{H}, s, \mathrm{H}-20), 1.56(3 \mathrm{H}, s, \mathrm{H}-38), 1.54(1 \mathrm{H}, m, \mathrm{H}-7), 1.30(3 \mathrm{H}, s$, $\mathrm{H}-23), 1.23(3 \mathrm{H}, s, \mathrm{H}-32), 1.14(1 \mathrm{H}, d d, J=13.9,13.7 \mathrm{~Hz}, \mathrm{H}-29), 1.07$ (3H, $s, \mathrm{H}-33), 1.05$ (3H, $s, \mathrm{H}-$ 22); ${ }^{13} \mathrm{C}-\mathrm{NMR}$ (pyridine- $d_{5}, 125 \mathrm{MHz}$ ): $\delta 207.9$ (C-9), 195.0 (C-4), 193.0 (C-10), 172.2 (C-3), 171.4 (C-2), 153.7 (C-14), 147.8 (C-13), 134.5 (C-19), 133.7 (C-36), 132.9 (C-26), 130.9 (C-11), 126.4 (C25), 124.3 (C-16), 122.8 (C-35), 121.7 (C-18), 116.6 (C-12), 116.5 (C-15), 87.2 (C-31), 69.2 (C-5), 52.2 (C-1), 47.0 (C-7), 46.8 (C-6), 43.8 (C-30), 39.8 (C-8), 30.4 (C-34, C-24), 29.4 (C-33), 29.1 (C29), 27.1 (C-22), 26.7 (C-17), 26.6 (C-20), 26.5 (C-27), 26.2 (C-37), 23.1 (C-23), 21.7 (C-32), 19.0 (C28), 18.8 (C-21), 18.3 (C-38); HREIMS [M-Na] ${ }^{+} m / z$ 625.3499, $\mathrm{C}_{38} \mathrm{H}_{50} \mathrm{O}_{6}$ requires 625.3505.

7-epi-Isogarcinol (5): Brown powder; $[\alpha]_{\mathrm{D}}^{25}=-158\left(c=1.0, \mathrm{CHCl}_{3}\right)$; UV $(\mathrm{MeOH}) \lambda_{\max }(\log \varepsilon): 319$ (3.85), 276 (4,15), 233 (4.12); IR $v_{\max }$ (ns) 3320, 2970, 2930, 1730, 1650, 1590, 1520, 1440, 1370, 1290, $1170 \mathrm{~cm}^{-1}$; ${ }^{1} \mathrm{H}$ - NMR (pyridine- $\left.d_{5}, 500 \mathrm{MHz}\right): \delta 8.13(1 \mathrm{H}, s l, \mathrm{H}-12), 7.72(1 \mathrm{H}, d l, J=7.7 \mathrm{~Hz}, \mathrm{H}-$ 16), 7.31 ( $1 \mathrm{H}, d l, J=7.6 \mathrm{~Hz}, \mathrm{H}-15), 5.38(1 \mathrm{H}, t l, J=6.2 \mathrm{~Hz}, \mathrm{H}-18), 5.18(1 \mathrm{H}, t l, J=6.2 \mathrm{~Hz}, \mathrm{H}-25)$, $5.13(1 \mathrm{H}, t l, J=6.6 \mathrm{~Hz}, \mathrm{H}-35), 3.28(1 \mathrm{H}, d d, J=13.9,3.2 \mathrm{~Hz}, \mathrm{H}-29), 2.97(1 \mathrm{H}, d d, J=13.6,6.4 \mathrm{~Hz}$, $\mathrm{H}-17), 2.77(1 \mathrm{H}, d d, J=13.6,4.8 \mathrm{~Hz}, \mathrm{H}-17), 2.44(1 \mathrm{H}, m, \mathrm{H}-7), 2.43(1 \mathrm{H}, m, \mathrm{H}-8), 2.26(1 \mathrm{H}, d d$, $J=14.1,2.5 \mathrm{~Hz}, \mathrm{H}-24), 1.96(1 \mathrm{H}, m, \mathrm{H}-34), 1.82(1 \mathrm{H}, d d, J=14.1,8.5 \mathrm{~Hz}, \mathrm{H}-24), 1.79(1 \mathrm{H}, m, \mathrm{H}-34)$, $1.77(1 \mathrm{H}, m, \mathrm{H}-8), 1.73$ (3H, $s, \mathrm{H}-21), 1.71(3 \mathrm{H}, s, \mathrm{H}-37), 1.65$ (1H, $m, \mathrm{H}-30), 1.62$ (6H, s, H-27, $\mathrm{H}-28), 1.56(3 \mathrm{H}, s, \mathrm{H}-38) 1.55(3 \mathrm{H}, s, \mathrm{H}-20), 1.27(3 \mathrm{H}, s, \mathrm{H}-23), 1.21(1 \mathrm{H}, d d, J=13.9,13.7 \mathrm{~Hz}, \mathrm{H}-$ 29), 1.14 (3H, $s, \mathrm{H}-32), 1.08$ (3H, $s, \mathrm{H}-33), 0.83$ (3H, $s, \mathrm{H}-22) ;{ }^{13} \mathrm{C}-\mathrm{NMR}$ (pyridine- $\left.d_{5}, 125 \mathrm{MHz}\right): \delta$ 207.3 (C-9), 194.9 (C-4), 193.2 (C-10), 170.8 (C-2), 153.7 (C-14), 147.9 (C-13), 134.2 (C-19), 133.7 (C-36), 132.9 (C-26), 131.0 (C-11), 129.1 (C-3), 124.4 (C-16), 123.8 (C-25), 122.9 (C-35), 122.1 (C18), 117.1 (C-12), 116.5 (C-15), 87.6 (C-31), 71.4 (C-5), 52.2 (C-1), 46.8 (C-6), 43.8 (C-30), 43.1 (C8), 42.2 (C-7), 30.4 (C-34), 29.2 (C-33), 28.5 (C-24, C-29), 26.2 (C-27), 25.9 ( C-17), 26.2 (C-37, C20), 22.8 (C-23), 21.7 (C-32), 18.8 (C-21), 18.4 (C-28), 18.3 (C-38), 16.6 (C-22); HREIMS [M-Na] $m / z 625.3519, \mathrm{C}_{38} \mathrm{H}_{50} \mathrm{O}_{6}$ requires 625.3505 .

\subsection{Biological Activities}

The extracts and compounds were tested against the chloroquine-resistant FcB1/ Colombia strain of Plasmodium falciparum in 96-well plates by measuring [3H]-hypoxanthine incorporation by parasite as previously described [23]. The growth inhibition for each compound concentration was determined by comparing the radioactivity incorporated in the treated culture with that in the control culture 
maintained on the same plate. The concentrations causing 50\% inhibition of parasite growth (IC50) were calculated from the drug concentration-response curves. Chloroquine ${ }^{\circledR}$ was used as a control compound.

The human diploid embryonic lung cells MRC-5 were seeded into 96-well microplates at 2000 cells per well. The cytotoxicity assays were performed according to a published procedure [24]. Taxotere ${ }^{\circledR}$ was used as a control compound.

\section{Conclusions}

A chemical investigation of Rheedia acuminata bark was carried out in the framework of a global investigation on French Guiana flora. This study showed that the bark contained a new xanthone, 1,5,6-trihydroxy-3-methoxy-7-geranylxanthone, together with 2-(1'-1'-dimethylprop-2'-enyl)-1,4,5trihydroxyxanthone. Pyrojacareubin, isogarcinol and 7-epi-isogarcinol were isolated from the Rheedia genus, for the first time. The two PPAPs isolated from Rheedia acuminata, which exhibited cytotoxic and antiplasmodial properties, were likely responsible of the biological activity found in the crude extract.

\section{Acknowledgements}

This work was supported by an ICSN-CNRS grant. We are grateful to Marie-Françoise Prévost (IRD) for her help in the identification of plant collection, Marie-Thérese Martin for her assistance in the structural determination and Geneviève Aubert (ICSN) who performed the cytotoxic assays on MRC-5 cells. All work carried out and reported in this manuscript is based on the program "Valorization of French Guiana flora" (CNRS, IRD, UMR Ecofog) with the financial support of the "Institut de Chimie des Substances Naturelles" (CNRS, France).

\section{References}

1. Grenand, P.; Moretti, C.; Jacquemin, H.; Prévost, M-F. Pharmacopées Traditionnelles en Guyane. Créoles, Palikur, Wayãpi; 2nd ed.; IRD: Marseille, France, 2004; pp. 309-319.

2. Peres, V.; Nagem, T.J.; Oliveira, F.F. Tetraoxygenated naturally occurring xanthones. Phytochemistry 2000, 55, 683-710.

3. Peres, V.; Nagem, T.J. Trioxygenated naturally occurring xanthones. Phytochemistry 1997, 44, 191-214.

4. Torrico, F.; Velasco, P.; Gimenez, A.; Almanza, G.R. An antibacterial xanthone and triterpenes of Rheedia acuminata. Rev. Boliv. Quim. 2001, 18, 38-42.

5. Cao, S.; Brodies, P.J.; Miller, J.S.; Ratovoson, F.; Birkinshaw, C.; Randrianasolo, S.; Rakotobe, E.; Rasamison, V.E.; Kingston, D.G.I. Guttiferones K and L, Antiproliferative compounds of Rheedia calicola from the Madagascar rain forest. J. Nat. Prod. 2007, 70, 686-688.

6. Li, X-C.; Joshi, A.S.; Tan, B.; ElSohly, H.N.; Walker, L.A.; Zjawiony, J.K.; Ferreira, D. Absolute configuration, conformation, and chiral properties of flavanone-( $3 \rightarrow 8$ ")-flavone biflavonoids from Rheedia acuminata. Tetrahedron 2002, 58, 8709-8717. 
7. Almeida, L.S.B.; Murata, R.M.; Yatsuda, R.; Dos Santos, M.H.; Nagem, T.J.; Alencar, S.M.; Koo, H.; Rosalen, P.L. Antimicrobial activity of Rheedia brasiliensis and 7-epiclusianone against Streptococcus mutans. Phytomedicine 2008, 15, 886-891.

8. Verdi, L.G.; Pizzolatti, M.G.; Montanher, A.B.P.; Brighente, I.M.; Smânia Júnior, A.; Smânia, E.D.F.A.; Simionatto, E.L.; Delle Monache, F. Antibacterial and brine shrimp lethality tests of biflavonoids and derivatives of Rheedia gardneriana. Fitoterapia 2004, 75, 360-363.

9. Zelefack ,F.; Guilet, D.; Fabre, N.; Bayet, C.; Chevalley, S.; Ngouela, S.; Ndjakou Lenta, B.; Valentin, A.; Tsamo, E.; Dijoux-Franca, M-J. Cytotoxic and antiplasmodial xanthones from Pentadesma butyracea. J. Nat. Prod. 2009, 72, 954-957.

10. Han, A.R.; Kim, J.A.; Lantvit, D.D.; Kardono, L.B.S.; Riswan, S.; Chai, H.; De Blanco, E.J.C.; Farnsworth, N.R.; Swanson, S.M.; Kinghorn, A.D. Cytotoxic xanthone constituents of the stem bark of Garcinia mangostana (mangosteen). J. Nat. Prod. 2009, 72, 2029-2031.

11. Pattalung, P.; Thongtheeraparp, W.; Wiriyachitra, P.; Taylor, W.C. Xanthones of Garcinia cowa. Planta Medica 1994, 60, 365-368.

12. Lee, H-H.; Chan, H-K. 1,3,6-Trihydroxy-7-methoxy-8-(3,7-dimethyl-2,6-octadienyl)xanthone from Garcinia cowa. Phytochemistry 1977, 16, 2038-2040.

13. Sordat-Diserens, I.; Marston, A.; Hamburger, M.; Hostettman, K. Novel prenylated xanthones from Garcinia gerrardii Harvey. Helv. Chim. Acta 1989, 72, 1001-1007.

14. Monach, G.D.; Monache, F.D.; Waterman, P.G.; Crichton, E.G.; De Limas, R.A. Minor xanthones from Rheedia gardenaria. Phytochemistry 1984, 23, 1757-1759.

15. Harrison, L.J.; Leong, L.S.; Sia, G.L.; Sim, K.Y.; Tan, H.T.W. Xanthones from Garcinia forbesii. Phytochemistry 1993, 33, 727-728.

16. Marti, G.; Eparvier, V.; Moretti, C.; Susplugas, S.; Prado, S. ; Grellier, P.; Retailleau, P., Guéritte, F.; Litaudon M. Antiplasmodial benzophenones from the trunk latex of Moronobea coccinea (Clusiaceae). Phytochemistry 2009, 70, 75-85.

17. Ciochina, R.; Grossman, R.B. Polycyclic polyprenylated acylphloroglucinols. Chem. Rev. 2006, 106, 3963-3986.

18. Hamed, W.; Brajeul, S.; Mahuteau-Betzer, F.; Toison, O.; Mons, S.; Delpech, B.; Hung, N.V.; Sevenet, T.; Marazano, C. Polyprenylated benzoylphloroglucinol derivatives from Garcinia oblongifolia. J. Nat. Prod. 2006, 69, 774-777.

19. Azebaze, A.G.B.; Meyer, M.; Valentin, A.; Nguemfo, E.L.; Fomum, Z.T.; Nkengfack, A.E. Prenylated xanthone derivatives with antiplasmodial activity from Allanblackia monticola Staner L.C. Chem. Pharm. Bull. 2006, 54, 111-113.

20. Tao, S.J; Guan, S.H.; Wang, W.; Lu, Z.Q.; Chen, G.T.; Sha, N.; Yue, Q.X.; Liu, X.; Guo, D.A. Cytotoxic polyprenylated xanthones from the resin of Garcinia hanburyi. J. Nat. Prod. 2009, 72, 117-124.

21. Ignatushchenko, M.V.; Winter, R.W.; Riscoe, M. Xanthones as antimalarial agents: Stage specificity. Am. J. Trop. Med. Hyg. 2000, 62, 77-81.

22. Ignatushchenko, M.V.; Winter, R.W.; Bächinger, H.P.; Hinrichs, D.J. Riscoe, M.K. Xanthones as antimalarial agents; studies of a possible mode of action. FEBS Letters 1997, 409, 67-73. 
23. Frappier, F.; Jossang, A.; Soudon, J.; Calvo, F.; Rasoinevo, P.; Ratsimamanga-Urverg, S.; Saez, J.; Schrevel, J.; Grellier, P. Bisbenzylisoquinolines as modulators of chloroquine resistance in Plasmodium falciparum and multidrug resistance in tumor cells. Antimicrob. Agents Chemother. 1996, 40, 1476-1481.

24. Tempete, C.; Werner, G.H.; Favre, F.; Rojas, A.; Langlois, N. In vitro cytostatic activity of 9-demethoxyporothramycin B. Eur. J. Med. Chem. 1995, 30, 647-650.

Sample Availability: Samples of the compounds are available from the authors.

(C) 2010 by the authors; licensee MDPI, Basel, Switzerland. This article is an open access article distributed under the terms and conditions of the Creative Commons Attribution license (http://creativecommons.org/licenses/by/3.0/). 